\title{
RFID: untangling mutant phenotypes
}

\author{
Lina Zeldovich
}

Improvements to RFID technology are making it an increasingly flexible tool for tracking genetically modified rodents, characterizing their natural behaviors, and improving data integrity.

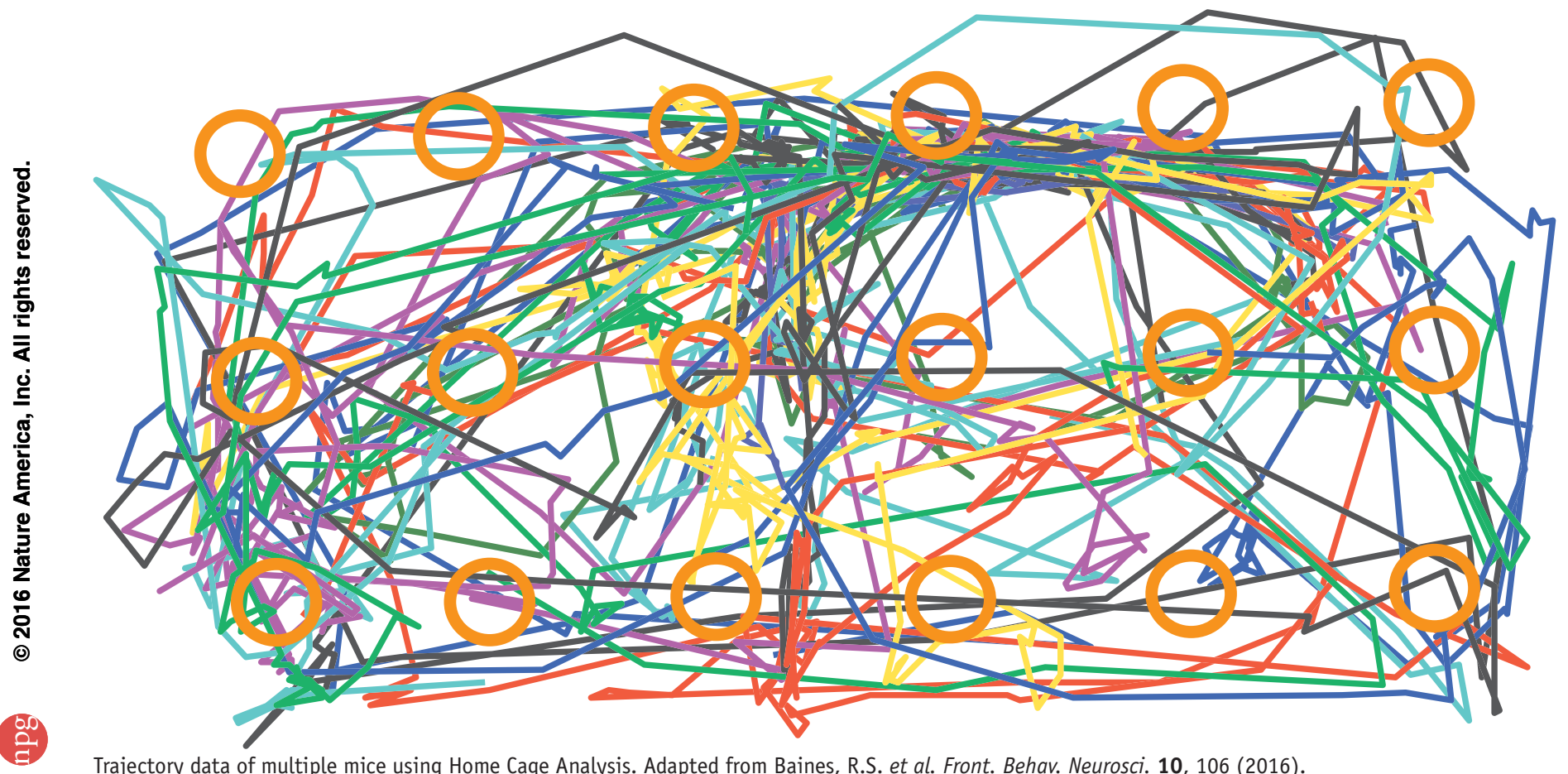

It was an ordinary task, but they were doing it in an unusual way. Mouse caretakers at MRC Harwell, an International Centre for Mouse Genetics in Oxfordshire, United Kingdom, were checking on their study subjects-a mice trio dwelling in their homecage. However, rather than regularly peeking in the cage, the caretakers were using a high-tech form of eavesdropping, dubbed Homecage Analysis (HCA.) HCA is a research apparatus the size of a typical individually ventilated mouse cage (IVC), but capable of providing far more information about the animals it houses. With a grid of 18 radiofrequency readers embedded underneath, and an infrared video

Lina Zeldovich is an editor and journalist. Correspondence should be addressed to L.Z. (linazeldovich@gmail.com) camera occupying the slot next to it on the rack, HCA provides around-the-clock information about animal movement and location that would be unfeasible to collect manually by staff and researchers.

But to bring out the full potential of HCA, there's another crucial ingredient: the mice themselves. At MRC Harwell, they weren't just any ordinary transgenic rodents. Each had a tiny electronic chip, known as a radiofrequency identification (RFID) tag, embedded underneath its skin, which essentially "wired" the mice into the HCA rig. The tagged mice could move around their home while the radiofrequency readers below detected the chips' locations, capturing the rodents' whereabouts ${ }^{1}$. "It's like a system of coordinates," says Douglas Armstrong, professor of systems neurobiol- ogy at the University of Edinburgh, UK, one of the study authors. "Effectively what we get from the system is the approximate location [of the mice] every second." For mice, it removes the unpleasant presence of humans during checkups, which causes stress and disrupts the rodents' normal behavior.

Designed in collaboration with the National Council of 3Rs, a UK-based organization that spearheads new technologies to replace, reduce and refine the use of animals for scientific purposes, the HCA system is an example of researchers using RFID technology to help solve the escalating challenges of managing and studying mouse colonies. The efficacy and reliability of modern biomedical research relies on transgenic mice-the workhorse of most in vivo studies. But while the numbers of animals 


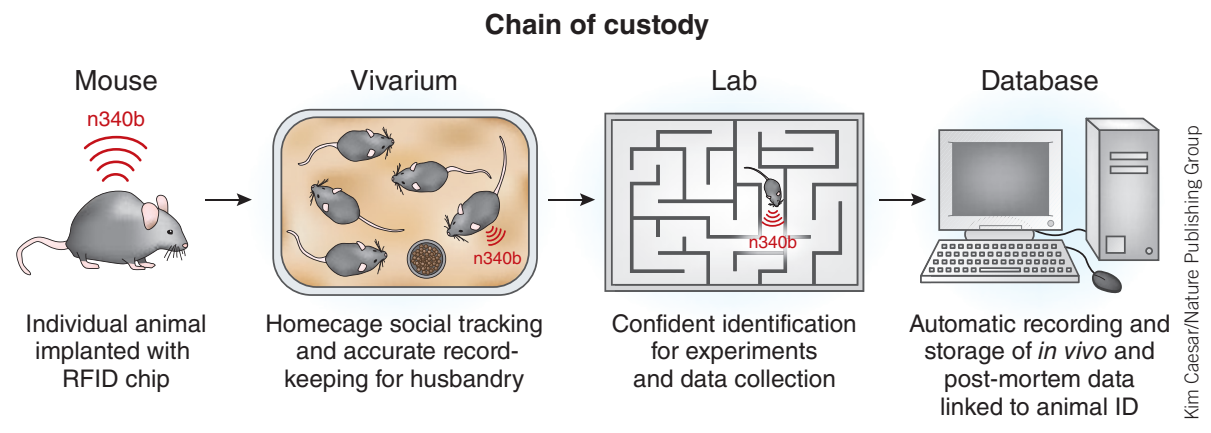

and the complexity of research have both increased-for example, the International Mouse Phenotyping Consortium plans to generate a knockout line for every mouse gene $^{2}$ - the traditional methods of tracking rodents and their behavior have not kept up.

Labs are calling for better methods of identifying and tracking mice, as well as smarter, less invasive tools to study them. Some researchers in academia and commercial drug development are turning to the RFID approach, which potentially addresses two issues with one solution: keeping track of the mice's identities and enabling ways to study their natural behavior without human interference.

\section{Social tracking}

Mice are social creatures, but observing and quantifying their group activity can be difficult. As a prey species with a history of being eaten by just about every predator imaginable, they are understandably skittish. The presence of a researcher or caretaker can be very disruptive; like a giant crashing a tea party. An additional challenge is that mice come out to play at night, when most scientists and staff have gone home. Automated methods for tracking behavior can help overcome these challenges.

When Armstrong's team left mice undisturbed in their natural group settings for long periods of time, they discovered new aspects about the social behavior of these animals. For example, while the team found that mice were active in the dark and mostly inactive and huddled together with lights on-as would be expected for nocturnal creatures-the mice also showed "bouts of activity" even with lights on ${ }^{1}$. These findings contrast with data from most studies that rely on running wheels to assess rodents' daily movements. Another interesting finding was that mice were able to anticipate the approaching "lights off" time; they became more active and started moving away from each other. Study authors noted that this was a peculiar circadian clock feature that wheel setups don't capture.

Because it is non-invasive-aside from an initial surgical implantation-RFID technology offers an improved method to study rodent natural behavior and social interactions. Mice serve as models for many neurodegenerative and central nervous system disorders, such as autism and Huntington's disease. To study such conditions, researchers often put mice through a battery of tests that require removing animals from their homecages, which can stress them and muddy up study results. In addition, isolating mice to understand disorders with social manifestations might not provide the most

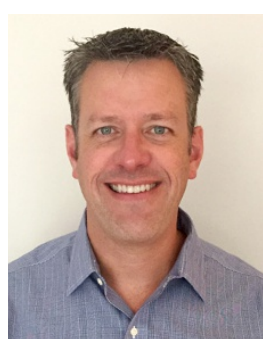

RFID chips can be used to track more than just an animal's identity, says Matt Ruiter. meaningful results; without playmates, studying social behavior is impossible. The ability to observe rodents' group activity without human intervention would make data from such studies more realistic and valuable.

\section{Chip technology}

RFID chips, also called transponders, don't carry any batteries. They are remotely activated by readers, also called scanners, via radiofrequency waves. When activated, the chips beam back data they are programmed to return-a unique identifier, such as a name or a number, and possibly some other information. Since the 1990s, veterinarians have widely used this technology in tagging pets. But in mice, adop- 
tion of this technology has been slower.

Jeffrey Everitte at Duke University, who worked with various types of RFID transponders throughout his career, says that a price tag of a few dollars per chip per mouse, plus at least two readers-a primary and a backup - added extra cost not every lab was willing to pay. Another concern was animal welfare. "For transgenic mice you gotta be able to identify very young mice," Everitte says - and the early chips were simply too big for the little pups. Implanting a transponder required a large needle, which could hurt the animal, he says. "The major impediment to the wide use of transponders was that they were relatively large for small mice."

Today's transponders come in a variety of sizes-many small enough to use in mice-and while their cost still typically ranges from $\$ 3$ to $\$ 7$ a chip, the price tag seems more acceptable as the technology helps researchers gather data they couldn't get before, manage their colonies, and feel confident they won't make mistakes.

While some chips can only be read, others can also be written on. UID Identification Solutions manufactures a writable chip that not only returns a unique number, but also lets users write important specific data about the mouse onto it-for example the name of its mutant gene. "Our technology is getting the identity of the animal and putting it into a useful format researchers can do something with," explains company president Matt Ruiter. The company also provides users with software that can streamline and error-proof important aspects of study procedures using RFID. For example, with drug studies that require a series of blood tests, the technicians can scan the mouse and then scan the bloodvials to assure they have the right materials for the right animal. "We can scan a blood tube and through our software we can say this blood tube matches this animal and this is the right time for it," Ruiter says. His firm plans to develop this concept further to let customers scan catheters, test tubes and other paraphernalia, information that can be helpful for large-scale operations. With UID software, mouse identity can live on even after the animal has been euthanized; its tissue slides can still be tracked by the same unique identifier it had while it was alive.

\section{RFID and reproducibility}

Another problem RFID technology addresses is misidentification of mice during experiments, which can affect the reproducibility of research results. Today, many labs use manual methods for marking rodents and

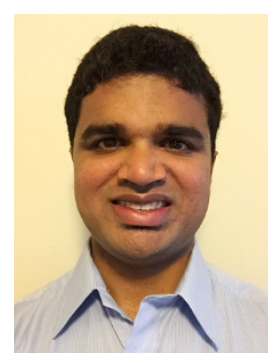

Studying mice under more natural nocturnal conditions creates challenges for accurate identification, says Krsna Rangarajan.

identification system.

All the traditional identification tools, from sharpie markers to ear tags to tattoos, have flaws. Mice are voracious groomers, so sharpie marks stay for a day or two and must be re-applied two to three times a week, Arlund says. That process is not only labor-intensive, but also problematic-for example marks can fade while lab vets are away for a long weekend. "It only takes one character to fade and I can't tell number 17 from number 18," Arlund explains. That ambiguity can cause significant problems for certain studies. Other methods are prone to issues too. Ear tags can fall off; ear punches, which have patterns that act as codes for identifying specific animals, can be confusing; and even permanent tail tattoos can be misread.

Most identifiers can't be read in the dark, which can be a requirement to study the natural behavior of mice. If the mice get mixed up, researchers often have to discard them and restart the study, wasting animals, time and money. "Obviously there's a problem that needs be solved," says Arlund. "Old fashioned methods of identifying animals in a breeding colony and animals in a study need to be improved."

Although not garnering as much attention as other sources of variability, animal misidentification is an obvious problem for reproducibility. "One of the components that seem to make up the lack

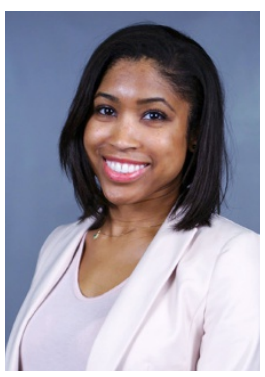

of reproducibility is identification and control of subjects," Arlund says. While Somark's permanent tail tattoos don't fade, technicians still can misread them, especially with multiple mice in a cage. Somark is now implementing an RFID system that will use chips and scanners similar to Armstrong's setup-

sing RFID tags to track an animal's pedigree can help with breeding transgenic colonies, says Meredith Dixon. in addition to tattoos. When scanned, the
chips will provide the technician with the animal's unique identifier, reducing, and hopefully eliminating, human errors.

For Krsna Rangarajan, who studies vision and attention in mice at the National Institute of Health, P-chips by PharmaSeq Inc do the job. Rangarajan puts his mice through tests in a specially designed behavioral apparatus, in which the animals get baby formula if they pay attention and lick the proper spout. To let the mice exhibit their natural nocturnal behavior, the tests must be completed in the dark, which increases the possibility of mistakes. "This makes it difficult for me, the operator, because I have to move the animals around in a dark room and put them into the behavioral apparatus," Rangarajan says. "We thought in advance this is a potential problem that I can get animals mixed up in different cages, so in addition to tattoos we also wanted another unique identifier." P-chips are only 500 microns wide-one of the smallest chip-types available - and are easily implanted into mice's tails. However, P-chips are not radiofrequency devices. They don't operate on radio waves, but instead use light: when scanned under a small laser, they beam back the rodent's number for the researcher to visualize. The process can be done under dark-room conditions. Before testing, Rangarajan scans his subjects' tails under the laser to verify he has the right critter. "This system makes me feel extra confident that I will not get my animals crossed," he says.

\section{Benefits for breeding}

When coupled with database software, RFID technology can help scientists and technicians manage a large mouse colony. 
Information and data about specific mice are organized and can be quickly retrieved by veterinarians, investigators and staff. Meredith Dixon, a research assistant at ALS Hope Foundation, uses RFID technology to keep track of about 600 mice of 31 different strains that are used for motorneuron disease studies. Her team breeds mice with mutant genes and assesses the progress of diseases. Mice are phenotyped and implanted with chips made by a company called AVID. Each mouse is assigned a unique number, which not only helps Dixon find the right animal in the right cage for assessment, but it also serves as a database record that links the mouse's data, including its genetics and family lineage. These records come in handy for breeding, Dixon says. "We put all mice that are born and microchipped into a big database," she explains. "We document who is paired with who and how many pups they have." By scanning an animal, Dixon can quickly find out who its parents or siblings were.

\section{Future prospects for disease studies}

Some pharmaceutical companies want to incorporate RFID technology into a variety of studies on many diseases, including cancer, cardiovascular disease and psychiatric disorders. One feature that RFID chips can help with is telemetry - the ability to read a mouse's temperature, heart rate or other vitals remotely, without human intervention. This can be very helpful in toxicology studies, in which the animals are dosed with a particular drug for several days while researchers observe whether mice develop a fever, an eventual tolerance to the drug, or specific side effects. Manually measuring parameters like body temperature would require significant amounts of human handling; a nuisance that chips can help avoid.

While the HCA system used by Armstrong and MRC Harwell provides a proof of concept, it opens up many prospects for biomedical studies. "The primary goal of that study was to see if the system works," Armstrong says, "But we now want to move on to look at disease mutants, drug effects, and so on."

1. Baines, R.S. et al. Front. Behav. Neurosci 10, 106 (2016).

2. Brown, S.D. \& Moore, M.W. Dis. Model. Mech. 5 289-292 (2012). 